\title{
THE RADIO REFERENCE FRAME OF THE U.S. NAVAL OBSERVATORY RADIO INTERFEROMETRY PROGRAM
}

\author{
T.M. Eubanks, M.S. Carter, F.J. Josties, \\ D.N. Matsakis and D.D. McCarthy \\ U.S. Naval Observatory \\ Washington, D.C., 20392-5100 \\ U.S.A.
}

\begin{abstract}
The U.S. Naval Observatory Navnet program monitors changes in the rotation of the Earth on a regular basis using radio interferometric observations acquired with telescopes in Alaska, Hawaii, Florida, West Virginia and, in the past, Maryland; other radio telescopes have also participated occasionally. These observations have been used to derive a radio interferometric celestial reference system, Navy 1990-5, using two years of dual frequency measurements from 24-hour-duration observing sessions. A total of 84 extragalactic radio sources, mostly quasars, have been observed by the Navnet program to date, of which 70 currently have source position formal errors of one milli second of arc or less. The root mean square of the difference between source position estimates from the Navnet data and an independently derived catalog using completely different data is less than one milli second of arc in both right ascension and declination after the adjustment of an arbitrary rotational offset between the two celestial reference frames.
\end{abstract}

\section{Introduction}

As part of its participation in the National Earth Orientation Service (NEOS) the U.S. Naval Observatory (USNO) operates the Navnet program in Very Long Baseline Interferometry (VLBI) to monitor changes in the orientation of the Earth on a regular basis. NEOS is a joint cooperative effort of the USNO and the National Geodetic Survey (NGS), and this VLBI program is designed to complement the observations coordinated by the NGS as part of the International Radio Interferometric Surveying (IRIS) subcommission. The Naval Research Laboratory (NRL) and the Crustal Dynamics Project VLBI group at the National Aeronautics and Space Administration Goddard Space Flight Center (GSFC) have also assisted in the development of the program. The USNO program measures the orientation of the Earth in space from interferometric observations acquired with telescopes at Gilmore Creek, Alaska, Kokee Park, Hawaii, Richmond, Florida, Green Bank, West Virginia and, in the past, Maryland Point, Maryland. Radio telescopes at Mojave, California, Westford, Massachusetts, Algonquin Park, Canada, and Medicina, Noto, and Matera, Italy, have also participated in these experiments on an occasional basis. Current Navnet operations consist of one 24-hourduration observing session per week together with three-hour duration observing sessions on two other days of the week. The Navnet data are used to estimate UT1, polar motion and nutation for inclusion in the International Earth Rotation Service (IERS) combined solutions.

One 24-hour-duration experiment was observed in September, 1988; monthly 24hour Navnet experiments commenced in April, 1989, and all of the weekly Navnets have 
been 24 hours in duration since June 27, 1989. At present, one 24-hour Navnet experiment is scheduled every week using at least three of the Florida, Alaska, Hawaii and West Virginia antennas. These data are reduced and released to NEOS and the IERS on a regular basis; typical processing times are now on the order of 8 to 14 days from the acquisition of data to release of the final Earth orientation results.

The Navnet VLBI data are acquired using the Mark III VLBI data acquisition system as described by Clark et al. (1985). The Navnet VLBI data have all been correlated at the Washington Correlator and reduced and processed using the GSFC Calc and Solve computer program package. After correlation, fringe fitting, and the removal of any remaining bandwidth synthesis delay ambiguities, the data are used in a series of weighted least-squares solutions to define a Navnet VLBI reference frame and to estimate the Earth orientation within that reference frame. The data are processed with IERS standard models to the maximum extent possible (see IERS Technical note number 3 ). Unmodeled variations in the tropospheric propagation delays and the relative time offset between the station clocks are a significant source of error in geodetic VLBI. The surface pressure, temperature and relative humidity are recorded at each station and used to estimate the variations in the hydrostatic zenith tropospheric propagation delay. Further variations in these quantities are treated by the estimation of piecewise linear models directly in the least squares solutions. A new piecewise linear function is introduced every 60 minutes for the zenith tropospheric propagation delay and every 90 minutes for the station clocks.

\section{The Navnet Radio Reference Frame}

An attempt is made to align the Navnet reference frame as closely as possible with both

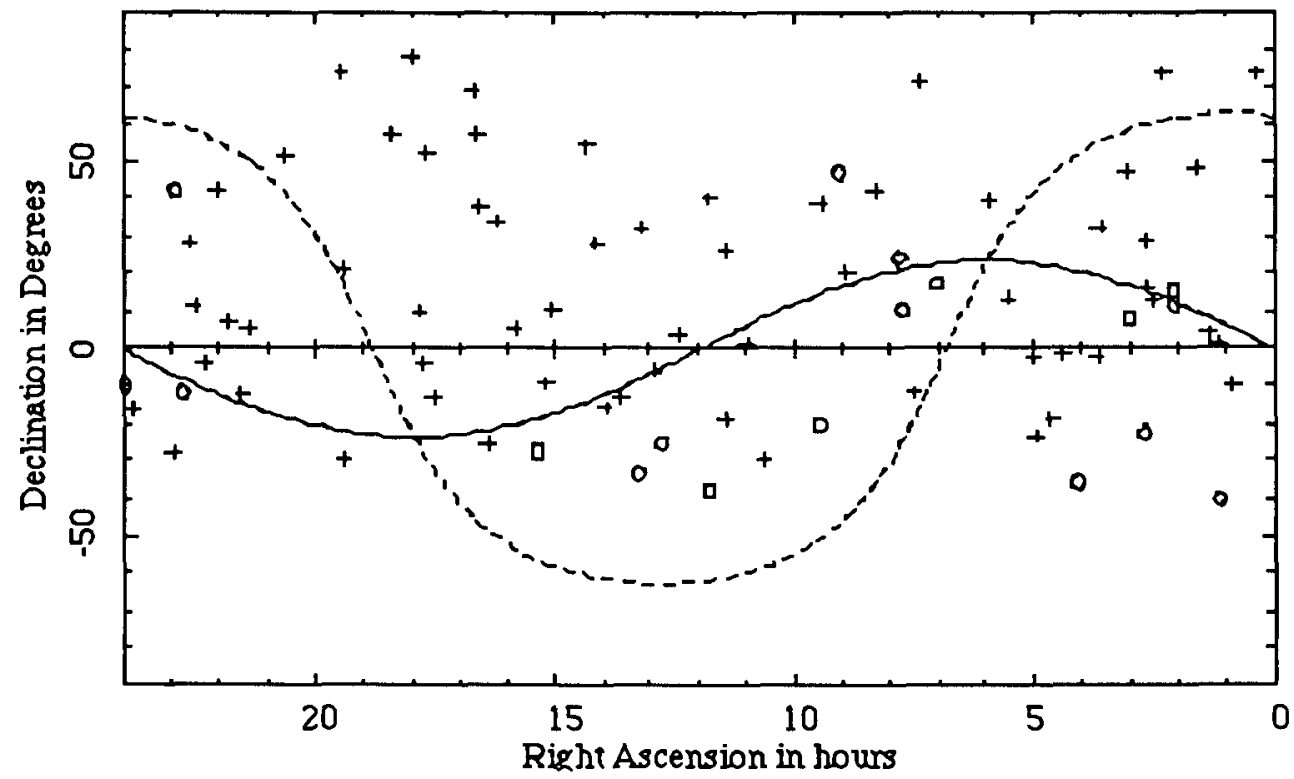

Figure 1 : The location in the sky of the sources currently used in Navnet operations. The primary sources used in the comparison with the GSFC GLB 353 catalog are indicated with crosses, other sources with open circles. The ecliptic and the galactic equator are denoted by the solid and dashed lines, respectively. 
the terrestrial and celestial reference frames maintained by the IERS, and to minimize bias offsets between the Navnet Earth orientation parameter estimates and those of the IERS combined solutions. Due to software limitations, the Navnet reference frame is obtained from the USNO VLBI data in a two solution process. In the first solution, the IERS Rapid Service (Bulletin A) estimates of the UT1 and polar motion are treated as a priori measurements and the nutation in longitude and obliquity is fixed at the IERS

Rapid Service values, while the Right Ascension ( $\alpha$ ) of the source 2216-038 is fixed at the value given in the IERS combined celestial reference frame for 1989 (R(IERS) $89 \mathrm{C}$ 01). All other source coordinates, and all of the station coordinates except for the Richmond station, were adjusted in this solution. The purpose of this solution is to align as closely as possible the Navnet celestial reference frame with the celestial pole implied by the IERS nutation series. The coordinates of two sources, $0202+149$ (in both $\alpha$ and

Declination, $\delta$ ) and $0742+103$ (in $\delta$ only) are then fixed in a second solution to the values obtained in the first solution. This second solution globally adjusts the coordinates of all of the other sources and all of the station coordinates except for the Richmond station, together with a separate adjustment for UT1, Polar Motion, both components of nutation and piecewise linear clock and troposphere models for each experiment. The IERS Rapid Service estimates for UT1 and polar motion are used as a priori measurements with weights as given by the IERS formal errors, but the two components of nutation are estimated freely for each observing session. The position of the Richmond station at the epoch 1988.0 is fixed at the value given by the ITRF- 88 for that epoch, and all stations are required to move at the AM0-2 rate. The USNO reference frame 1990-5 (IERS designation RSC (USNO) 90 R 05) was obtained from such a solution using all of the Navnet VLBI data from 67 24-hour-duration observing sessions from September 10, 1987 , through September 20, 1990; this solution used a total of 29216 observation pairs

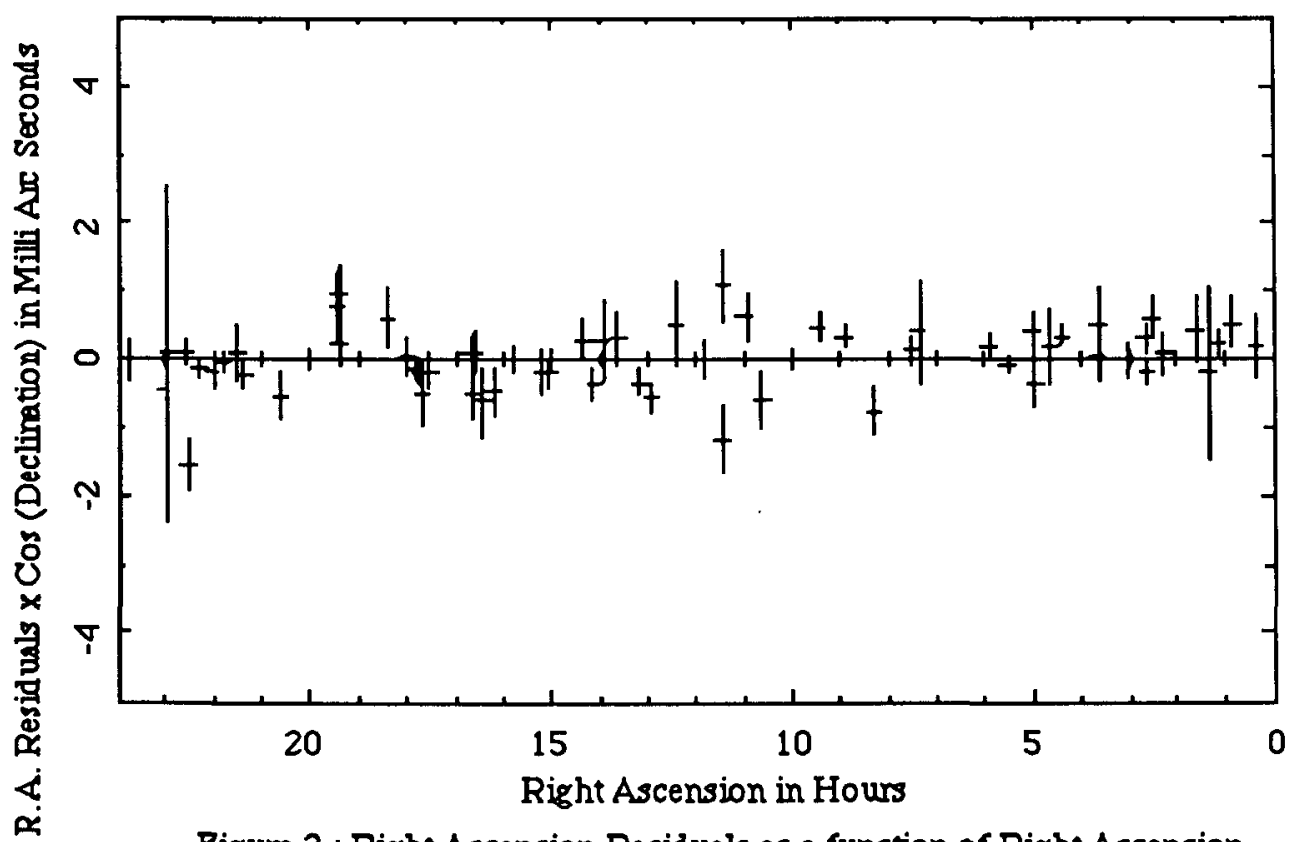

Figure 2 : Right Ascension Residuals as a function of Right Ascension 
(delay and delay rate) with a weighted rms residual scatter of \pm 49.7 picoseconds for the delay data and \pm 104.2 femtoseconds per second for the delay rate data. A total of 84 sources have been observed by the Navnet program; four radio sources $(3 \mathrm{C} 84,3 \mathrm{C} 273 \mathrm{~B}$, $3 \mathrm{C} 345$ and $3 \mathrm{C} 454.3$ ) were judged to have too much source structure to be usable for geodetic work and are no longer routinely observed; these sources were treated as "arc" parameters in the solution, with a separate position being estimated for each experiment in which they were observed. The Navy 1990-5 celestial reference frame thus consists of the positions of 80 radio sources, including the two reference sources, 68 sources with formal uncertainties of less than one mas in both components of position ("primary" sources), and 10 others with worse position estimates. The distribution of these sources in the sky is shown by Figure 1.

\section{Comparison of Independent Radio Reference Frames}

Several groups have derived Radio Interferometric reference frames using VLBI observations from transcontinental and intercontinental baselines. (see, e.g., Robertson et al., 1986, Sovers et al., 1988, and Ma et al., 1990). Reference frames derived from modern VLBI observations usually claim positional uncertainties on the order of 0.1 to 1 milli arc seconds (mas). Robertson et al. (1986), using a variety of internal repeatability checks, showed that the precision (but not necessarily the accuracy) of the Mark III derived NGS catalog is in the order of 0.5 mas or less. Sovers et al. (1988) and Ma et al. (1990) compared catalogs derived from Mark III observations with a catalog from the less sensitive JPL geodetic Mark II system and found root mean square (rms) agreement at the 2 mas level. Sub milli second of arc agreement has been found between various GSFC and NGS catalogs (see, e.g., the IERS Annual Reports for 1988 and 1989), but these catalogs, although independently processed, share a considerable amount of common data (all of the NGS IRIS data are used in the GSFC solutions), and thus these

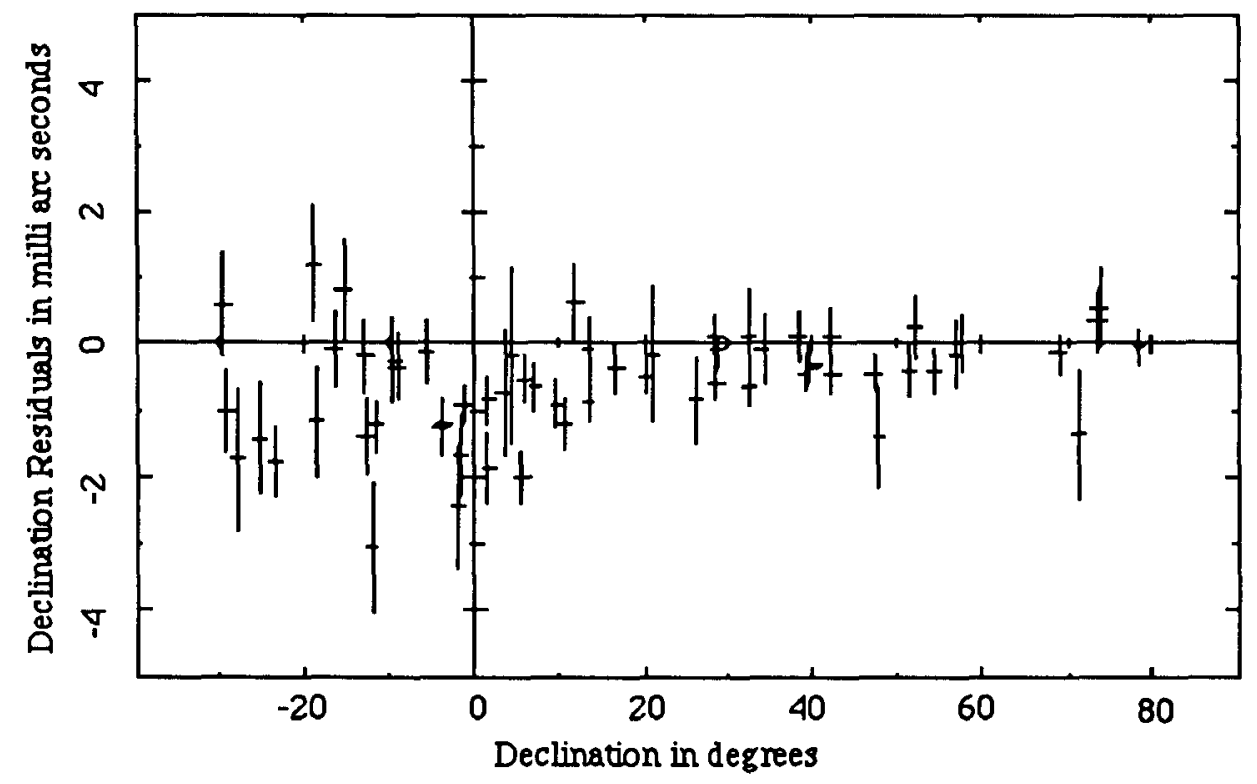

Figure 3 : Declination Residuals as a function of Declination 
comparisons are not a true measure of the accuracy of these catalogs (Arias et al. 1988).

Recently, Ma et al. (1990) published a catalog based on previous GSFC and NGS geodetic observations plus the first results of a GSFC/NRL/USNO program to determine a dense celestial reference frame using radio sources with optical counterparts. This catalog, denoted GLB 353, contains estimates of the positions of 182 sources including 62 of the 68 primary Navnet sources. The GLB 353 positions were subtracted from the Navy 1990-5 positions and an arbitrary rotation offset removed by estimating the A1, A2 and A3 parameters described in Arias et al. (1988). The agreement in $\alpha$, shown in Figure 2 , was excellent, with an rms scatter of only 0.46 mas after scaling the $\alpha$ residuals by the cosine of the declination, and very little evidence for systematic (non-random) patterns in the residuals. The scatter in the $\delta$ residuals is larger, with an ms value of 0.97 mas. The scatter (Figure 3) clearly increases towards lower declinations, probably a result of the smaller coverage in hour angle available for those sources from a network in the Northern hemisphere. The correlation, -0.25 , between the $\alpha$ and $\delta$ residuals is small and not significant.

The scatter between the Navy 1990-5 and the GSFC GLB 353 source positions, although small, is significantly larger in both components than would be expected on the basis of the formal errors provided by the two solutions. This effect was modeled by estimation of a constant additive variance; separate constants were estimated for the $\alpha$ residuals and $\delta$ residuals. The square root of these constants (the "additive noise") is 0.1 mas for the $\alpha$ residuals (again scaled by the cosine of the declination) and 0.55 mas for the $\delta$ residuals. The larger additive noise required for the $\delta$ residuals is associated with the increased residual scatter at declinations less than $10^{\circ}$, and may be related to failures in the adopted troposphere propagation delay model at low elevation angles, since the sources in the extreme South must be observed at low elevation angles from networks in the Northern hemisphere.

\section{Conclusions}

The Navnet celestial reference frame agrees with independent radio source position estimates at the level of one milli second of arc in declination and one half milli second of arc in right ascension. There is evidence that the models and procedures currently used may not be adequate at low declinations. The Navnet program continues to acquire weekly measurements and thus the Navnet observations will play an increasingly important role in the determination of the celestial radio reference frame.

\section{References}

Arias, E.F., Feissel, M., Lestrade, J.F.: Astron. Astrophys., 199, 357-363.

Clark, T.A., et al.: 1985, IEEE Trans. Geosci. Remote Sensing, GE-23, 438-448.

International Earth Rotation Service, Annual Report for 1988: Observatoire de Paris.

International Earth Rotation Service, Annual Report for 1989 : Observatoire de Paris.

Ma, C., Shaffer, D.B., de Vegt, C., Johnston, K.J., Russell, J.L.: 1990, Astron. J., 99, 1284-1298.

Robertson, D.S., Fallon, F.W., Carter, W.E.: 1986, Astron. J., 99, 1456-1462.

Sovers, O.J., Edwards, C.D., Jacobs, C.S., Lanyi, G.E., Liewer, K.M., Treuhaft, R.N.: 1988, Astron. J., 95, 16471658 . 